\title{
Image processing in applied optics educational programs
}

\section{Robert Bunch}

Robert M. Bunch, "Image processing in applied optics educational programs," Proc. SPIE 2525, 1995 International Conference on Education in Optics, (13 October 1995); doi: 10.1117/12.224037

SDIE Event: SPIE's 1995 International Symposium on Optical Science, SPIE. Engineering, and Instrumentation, 1995, San Diego, CA, United States 
Image processing in applied optics educational programs

\author{
R. M. Bunch \\ Rose-Hulman Institute of Technology \\ Department of Physics and Applied Optics and Center for Applied Optics Studies \\ Terre Haute, IN 47803
}

\begin{abstract}
$\underline{\text { ABSTRACT }}$
The acquisition and processing of images has grown into a routine tool used in research and industry today. Image processing tools can also be employed by students to better understand the basic science of the phenomena that they are studying. Even though students may be able to visualize the physical phenomena of interest during a particular laboratory experiment they often find it difficult to record and quantify their experimental results. Fortunately, computer hardware along with cameras of sufficient resolution are now available to assist in the acquisition of images and sufficiently powerful image processing software is available to perform analysis and measurements on these images. The use of image processing and image acquisition in applied optics lab courses will be discussed.

An interdisciplinary program that incorporates image processing and computer graphics into our curriculum will also be discussed. Faculty members from the Physics and Applied Optics, Electrical and Computer Engineering, and Computer Science Departments pooled their resources to create an Imaging Systems Laboratory. We now offer an Imaging Systems Certificate to students of any major at Rose-Hulman who complete a sequence of courses and an imaging project.
\end{abstract}

Keywords: optics education, image acquisition, image processing

\title{
1. INTRODUCTION
}

One of the best educational aspects of optics is that it is a visual science. Almost every lab experiment in optics results in the formation of some type of image. For example, we observe images formed on a screen in geometrical optics experiments, interference patterns from sources, diffraction patterns from apertures, interferograms in an optical testing experiment, etc. Even though the optical phenomena of interest can be observed by the students during a lab experiment, the students often find it difficult to record and document the phenomena for analysis. The challenge in the experiment is to record the amount of light as a function of position in the image plane for subsequent analysis.

Photographing the image formed in an optics experiment is probably the most common technique used for recording an image. However, making spatial measurements from a photograph can be a time consuming and inaccurate process. Fortunately, some experiments 
can be performed using a single detector. Fiber optics experiments are good examples of this type where the amount of light emitted from a fiber is collected. ${ }^{1}$ Measuring and recording the irradiance as a function of position can be done by scanning a single detector through an image but the procedure is tedious and time consuming. Employing a linear array detector ${ }^{2}$ can help speed up the data recording but requires a system for retrieving the measured values from an oscilloscope or other data acquisition hardware. If a linear array detector is used without a data acquisition system a photograph of the oscilloscope screen must be made in order to have a hard copy for subsequent analysis of the data.

Over the past few years image processing and image manipulation techniques have become more widely available. These techniques are beginning to be employed by educators for astronomy and medical applications ${ }^{3}$ as well as basic physics ${ }^{4}$. The use of image acquisition hardware and software in research and industry has dramatically expanded. ${ }^{5}$ These systems are tailor made for incorporation into optics laboratories.

This paper discusses the use of image acquisition and image processing tools in the undergraduate applied optics lab courses at Rose-Hulman Institute of Technology (RHIT). The continuing development of a standard image acquisition and analysis experimental lab station for general use in optics labs will be described. Some examples of how images can be used for optics experiments will be given. Also, an interdisciplinary program that incorporates image processing and computer graphics into our curriculum will be discussed.

\section{IMAGE PROCESSING TOOLS IN OPTICS LAB COURSES}

Over the years the faculty developing applied optics labs at RHIT have concentrated on acquiring and equipping our optics labs with general purpose equipment that can be configured to perform lab experiments on traditional optical phenomena, measurements and techniques. Examples of a few of the experiments that the students currently perform are:

- Diffraction and Fourier Transforms

- Abbe Theory of Image Formation

- Polarization Phenomena

- Interferometry and Fringe Analysis

- Transmission Holography

- Radiometry of Extended Sources and Spectral Content of Sources

- Light Emitting Diode and Laser Diode Characteristics

- Optical Testing Using A Fizeau Interferometer and a Twymann-Green Interferometer

- Acousto-optic Light Modulator Applications

- Spherical Aberrations of Lenses

- Numerical Aperture and Spectral Attenuation Measurement of Optical Fibers

- Fiber Optic Displacement Sensors

- Dye Laser Characteristics 
Using a CCD camera and a frame-grabber in a computer to acquire the images and make them readily available for post-processing will provide a number of advantages. This will free the students from spending their time in the lab dealing with details of acquiring data and let them concentrate on understanding the experiments. Difficult measurements of the dimensions from images would be simple. Display of graphs and histograms from the images would be straightforward.

\subsection{Lab stations}

A PC platform was chosen for the standard image acquisition and analysis experimental lab station because of available software, compatibility of software and hardware, and computing power per unit cost. Each system is also equipped with a separate dedicated CCD camera, a frame-grabber, an integrated hardware and software image processing package that runs interactively in the Windows environment, and hardware and software to allow connection to the campus network. The output from the frame-grabber processor is displayed on a separate monitor. Each computer is on a moveable cart allowing greater flexibility in the organization of the lab setups.

\subsection{Optics experiments enhanced with image processing tools}

Image processing tools have been incorporated into several of the applied optics lab courses at Rose-Hulman. Below are descriptions of two simple examples of the use of image processing tools incorporated into traditional optics lab experiments. The first example illustrates how the images can be used to analyze magnification and aberrations by an optical system. The second example shows how enhancement techniques can be used along with line-scans across an image to perform simple interferometric analysis.

Figure 1 shows an image of a grid formed by a high-quality lens to use as a reference for measurement and calibration. Studying magnification by this single-lens system is simply a matter of changing the lens position and determining the positions where a magnified (minified) image is formed and then recording changes in the grid pattern. An image of the same grid was then formed by another (low quality) lens as shown in Fig. 2. Using the image processing software it is a simple matter to indicate and record points on the grid, grid spacings, etc. Deviations from a reference image can also be used to analyze the aberrations of the single lens system as in the Hartmann test ${ }^{6}$. 


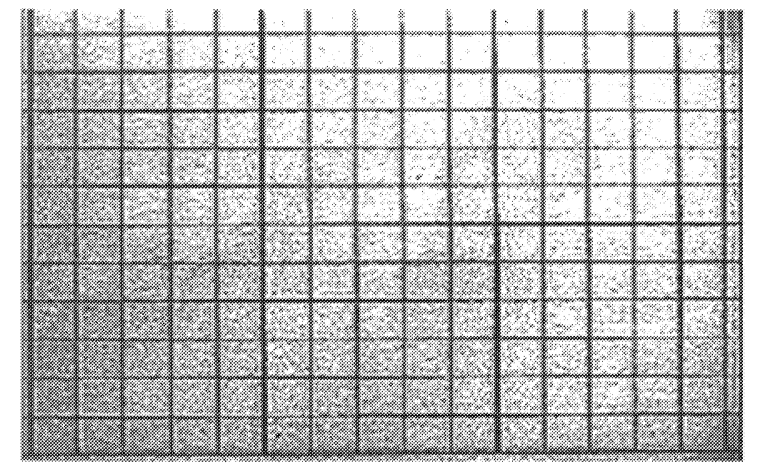

Figure 1 Reference grid image

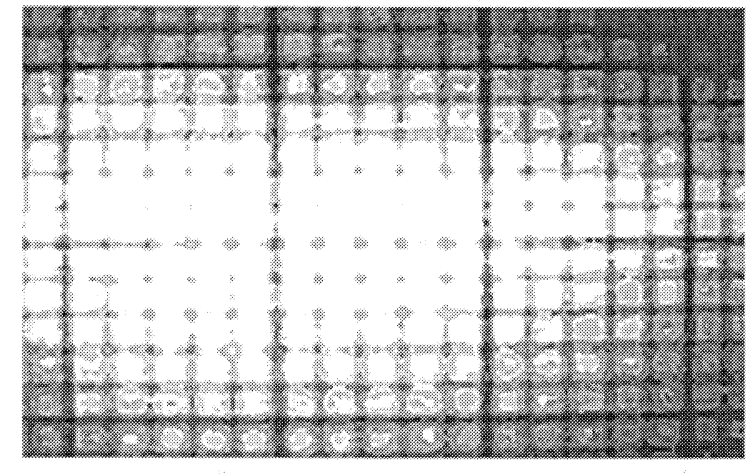

Figure 2 Image of a grid formed by a lens system under test.

The interferogram shown in Fig. 3 was obtained using a Twymann-Green interferometer configuration. The original image has poor visibility. By using histogram stretching techniques and median filtering, the interferogram was processed on-line to yield the image of Fig. 4. A line-scan tool provided by the image processing software can be used on this image to easily measure and count the fringes. The graph resulting from one of the linescans is shown in Fig. 5.

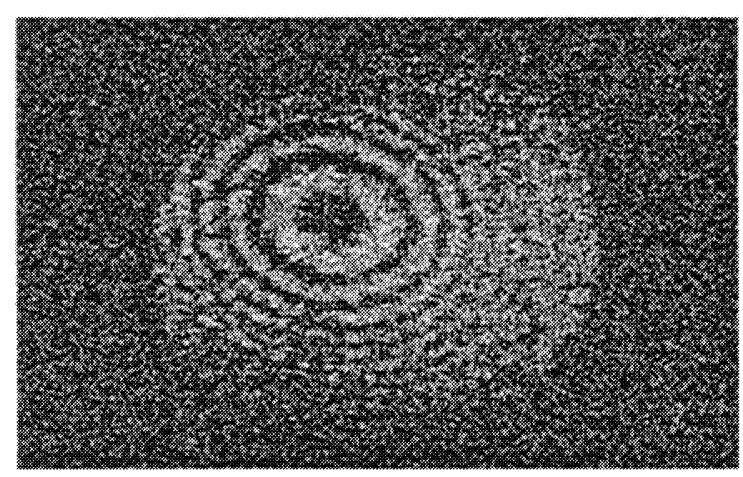

Figure 3 Interferogram from an optical testing experiment.

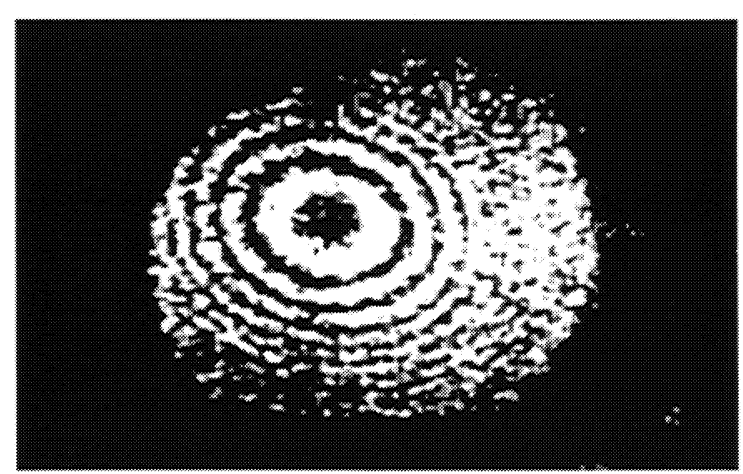

Figure 4 Resulting interferogram after processing. 


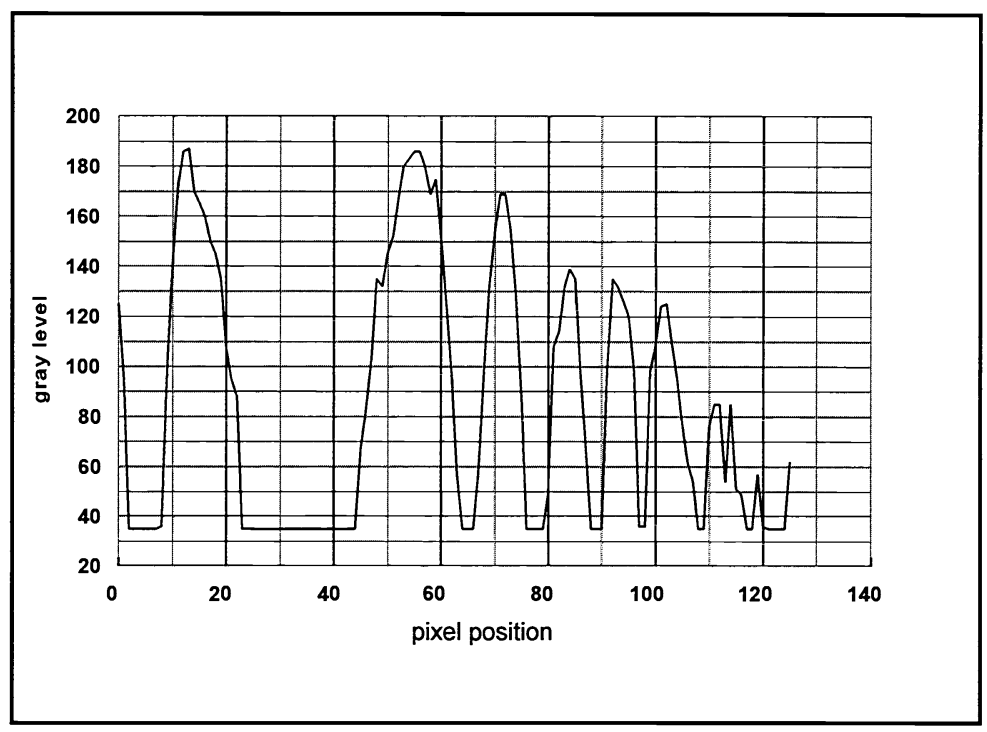

Figure 5 Line scan through a section of the interferogram image shown in Fig. 4.

The examples discussed are two of the more straightforward applications for using imaging processing hardware and software in applied optics lab. We have also employed similar imaging techniques to study diffraction, light emitting diode beam profile characteristics and numerical aperture of optical fibers. Additional applications for these tools will continue to develop as we become more familiar with the image processing hardware and software.

\section{IMAGING SYSTEMS CERTIFICATE PROGRAM}

Rose-Hulman Institute of Technology recently began offering an Imaging Systems Certificate that is a multidisciplinary educational program covering various aspects of image science and engineering. The certificate is intended to recognize undergraduate students who have gained a grounding in Imaging Systems through coursework and independent or teambased research on an imaging based project. The coursework provides students with a basic understanding of imaging areas including image processing and computer graphics. The project work attempts to give the student an opportunity to concentrate in some specific area of interest and utilize the knowledge gained from their courses. Currently there is no formal graduate program in imaging, however a number of graduate students in Applied Optics and in Electrical Engineering use the Imaging Systems Laboratory facilities and work on imaging projects for their thesis research work. 
Core Courses for the Imaging Systems Certificate:

EE437/PH437

EE537/PH537

CS351

Electives:

CS413

CS451

EE391

EE597/PH570

ME435

$\mathrm{PH} 280$

$\mathrm{PH} 292$

$\mathrm{PH} 380$

PH392

PH592
Introduction to Image Processing

Advanced Image Processing

Computer Graphics

\author{
Artificial Intelligence \\ Advanced Computer Graphics \\ Introduction to Communications Systems \\ Neural Networks \\ Robotics Engineering \\ Paraxial Optics \\ Physical Optics \\ Lens Design and Aberrations \\ Coherent Wave Optics \\ Fourier Optics and Applications
}

\section{CONCLUSION AND FUTURE DIRECTIONS}

Image acquisition and image analysis will play an increasing role in optics educational labs. At RHIT we will continue to develop experiments for out applied optics courses that utilize image acquisition and image processing tools. The examples discussed in this paper were only in the visible region of the spectrum. Several experiments could also be extended in to the near infra-red spectral region. With a relatively simple infrared viewer camera images of heated objects (wires, circuit boards, etc) can be acquired, analyzed and processed. Standard optical experiments such as the law of refraction can be demonstrated in the infrared region of the spectrum.

Incorporating the digital image acquisition lab station with optical image processing systems would also enhance the labs that accompany the courses required by the image processing certificate program. Hopefully, these tools will also assist the students with their imaging systems projects.

\section{ACKNOWLEDGMENTS}

This work was supported in part by National Science Foundation Instrumentation and Laboratory Improvement Program grants \#DUE-9350853 and the W. M. Keck Foundation. I would also like to thank Mr. Masood Makkar and Mr. Kelly Orr for assistance in acquiring the images used in this paper. 


\section{REFERENCES}

1. R. M. Bunch "Optical fiber sensor experiments for the undergraduate physics laboratory", Am. J. Phys. 58(9), 870-874 (1990).

2. James T. Wesley and Anthony F. Behof "Optical diffraction pattern measurements using a self-scanning photodiode array interfaced to a microcomputer", Am. J. Phys. 55(9) 835-844 (1987).

3. R. Greenburg, "Scanning the Images of Science" The Science Teacher, 59(8), 14-18, November 1992.

4. W. Benenson and W. Bauer "Frame grabbing techniques in undergraduate physics education", Am. J. Phys. 61(9) 848-851 (1993).

5. Laura Robinson, "In Imaging, What a Difference a Decade Makes" Photonics Spectra, 109-116, April 1995.

6. I. Ghozeil, "Hartmann and Other ScreenTests" Chapter 10 in Optical Shop Testing, 2nd Ed., pp. 367-396, Edited by D. Malacara, John Wiley \& Sons, New York (1992). 